INVESTIGACIÓN

\title{
FORMULACIÓN DE PROYECTOS PRODUCTIVOS DESDE EL AULA DE CLASE EN LAS INSTITUCIONES DE EDUCACIÓN MEDIA DE PITALITO - HUILA*
}

\section{FORMULATION OF PRODUCTIVE PROJECTS FROM THE CLASSROOM IN THE MEDITATION EDUCATION INSTITUTIONS OF PITALITO - HUILA}

\author{
Gloria Isabel Vargas-Hurtado*
}

Oscar Andres Benavides-Parra*

Recibido: 1 de marzo de 2018

Evaluado: 8 de mayo de 2018

Aprobado: 5 de julio de 2018

\section{RESUMEN}

El fomento del emprendimiento busca garantizar que los estudiantes de las Instituciones de Educación Media-IEM, cuenten con los espacios necesarios para desarrollar actividades de emprendimiento a partir de la identificación de necesidades reales de las comunidades y la generación de ideas innovadoras. Además, de motivar a los estudiantes para promover la formulación de proyectos productivos desde el aula como parte de su proceso de aprendizaje.

* Resultado del proyecto Investigación y emprendimiento, una estrategia de mejoramiento de la calidad educativa IEM del municipio de Pitalito con la articulación de la Educación media básica y superior. Artículo de investigación.

** Contador Público, Universidad de Ibagué. Especialista en Gestión Pública, Escuela Superior de Administración Publica. Magíster en Finanzas y Mercados Internacionales, Universidad de San Pablo CEU. Directora Zona Sur, CCAV Neiva, Universidad Nacional Abierta y a Distancia. Email: gloria.vargas@unad.edu.co. Contacto: 3124817001. https://orcid.org/0000-0003-4764-7613

$\star \star \star$ Administradora de Empresas, Universidad del Tolima. Especialista en Pedagogía para el Desarrollo del Aprendizaje Autónomo, Universidad Nacional Abierta y a Distancia. oscara.benavides@unad.edu.co. Contacto: 3013980755. https://orcid.org/0000-0001-7947-8047 
Por este motivo, se realiza una revisión documental iniciando con la normatividad, para luego contrastar lo definido por los autores referente al emprendimiento y la formulación de proyectos productivos en la educación media. Asimismo, se analiza la realidad actual y de las IEM de Pitalito, a partir del trabajo investigativo realizado con ocho Instituciones educativas de este municipio. Este artículo de reflexión, pretende identificar cuáles son las condiciones que se deben generar desde las IEM para dinamizar la formulación de proyectos productivos desde las aulas.

Palabras clave: Proyectos productivos, educación media, Pitalito, aula de clase, emprendimiento

\begin{abstract}
The promotion of entrepreneurship seeks to guarantee that the students of the Media Education Institutions-IEM, have the necessary spaces for the development of the identification of the needs of the communities and the generation of innovative ideas. In addition, motivation for students to promote the formulation of productive projects from the classroom as part of their learning process.
\end{abstract}

For this reason, a documentary review is carried out starting with the regulations, and then contrasts with the definition of the related projects in secondary education. In addition, the current reality and the IEM of Pitalito are analyzed, based on the research work carried out with eight educational institutions of this municipality. This article of reflection, aims to identify with the conditions that must be generated from the IEM to stimulate the formulation of productive projects from the classrooms.

Keywords: Productive projects, middle education, Pitalito, classroom, entrepreneurship

\section{INTRODUCCIÓN}

A partir de la Ley 1014 de 2006, que tiene como finalidad fomentar la cultura del emprendimiento, se establece entre los principios generales de su artículo 3, el "Fortalecimiento de procesos de trabajo asociativo y en equipo en torno a proyectos productivos con responsabilidad social" (p. 2), siendo uno de sus principios fundamentales la creación de nuevos productos interviniendo la realidad actual de las comunidades, pero a su vez siendo el resultado del emprendimiento que debe partir desde las 
aulas de clase, además se establece la enseñanza obligatoria en el artículo 13 de la ley citada, donde menciona que "los establecimiento de educación media deben definir un área específica de formación para el emprendimiento y la generación de empresas, la cual debe incorporarse al currículo y desarrollarse a través de todo el plan de estudios." (p. 6), al igual que armonizar los Proyectos Educativos Institucionales (PEI) con la Ley 115 de 1994 por la cual se expide la ley general de educación.

En consecuencia, el Ministerio de Educación Nacional-MEN (2012), proyecta la guía No. 39 que establece la cultura del emprendimiento en los establecimientos educativos, donde se orienta que:

“En el marco de la implementación de una cultura del emprendimiento, los proyectos pedagógicos son uno de los ambientes propicios para articular ideas innovadoras que respondan a necesidades identificadas en el contexto y constituyen una oportunidad para desarrollar prácticas enfocadas hacia el logro de un propósito colectivo." (p. 36).

En este sentido el emprendimiento debe evidenciarse desde lo curricular, planteando lineamientos claros para su implementación y desarrollo en las instituciones.
En este orden de ideas el emprendimiento debe ser tener un implementación significativa para lograr la generación de proyectos productivos que inician el desarrollo desde las aulas y buscan como producto final la generación de proyectos productivos que puedan intervenir en las comunidades, por lo tanto, el Ministerio de Educación Nacional-MEN (2010), establece el Proyecto Pedagógico Productivo-PPP:

"Como una estrategia educativa que ofrece a estudiantes, docentes e instituciones educativas, oportunidades para articular la dinámica escolar con la de la comunidad, considerando el emprendimiento y el aprovechamiento de los recursos existentes en el entorno, como una base para el aprendizaje $y$ el desarrollo social." (p. 10).

Esto permite que desde las aulas de clase se pueda involucrar a los estudiantes en el desarrollo de potencialidades para incentivar la creación de proyectos productivos que se ajustes a la realidad de las regiones, generando nuevo conocimiento y fomentando el emprendimiento desde de la educación media.

Sobre lo mencionado, Hernández (2006), menciona que "Una dinámica escolar con características emprendedoras, demanda la aplicación de métodos de enseñanza que promuevan la iniciativa del estudiante, la actividad creadora, la 
innovación escolar y el desarrollo del talento de los estudiantes en las tareas escolares y extraescolares." (p. 37), de acuerdo a lo mencionado, las instituciones deben fomentar el emprendimiento para obtener como resultado los proyectos productivos a partir de las experiencias de los alumnos. Asimismo, Santana, García \& González (2016) hace referencia sobre "la necesidad de trabajar la competencia para emprender, entendida como la capacidad de la persona para transformar las ideas en acción."(p. 136).

Con el propósito de promover la generación de proyectos productivos desde las aulas, el Ministerio de Educación articulado con las Secretarías de Educación y las Instituciones Educativas, también "ponen a disposición la Cartilla para el Desarrollo de Proyectos Pedagógicos Productivo y el manual para la formación que tiene como objetivo brindar orientaciones metodológicas que permitan instrumentar el ciclo de los PPP en las prácticas institucionales" (MEN, 2010. p.8), en este sentido, se busca que las instituciones incorporen los PPP articulados con los Proyectos Educativos Institucionales-PEI, no solo como una estrategia pedagógica para el aprendizaje, sino también como una herramienta que aporta a la innovación desde las aulas de clase para buscar soluciones en las comunidades, además de propiciar la generación de proyectos de vida.
Aparte de ello, Santana, García \& González (2016), considera que "La educación debe fomentar en los estudiantes: 1) la creatividad, la innovación y la asunción de riesgos, y 2) las habilidades para planificar, gestionar proyectos y conseguir los objetivos teniendo en cuenta los valores éticos." (p. 136), por lo tanto, las instituciones tienen el reto de fortalecer el fomento del emprendimiento, con la finalidad de generar ideas innovadoras, tomando decisiones que se ajusten a la realidad actual de las comunidades, aprovechando los recursos y necesidades de la región.

Del mismo modo, Novoa, A. (2004), hace referencia sobre "la interacción de docentes, alumnos, comunidad y escuela en el marco de los PPP organizado para conformar la dimensión productiva, que hace relación a los métodos, prácticas y recursos para producir bienes propios en su concepción ampliada" (p. 10), por lo tanto, es menester de las Instituciones de Educación Media-IEM, incorporar los PPP en las actividades curriculares y asegurar los espacios que propicien formas eficaces para mejorar de forma progresiva las competencias de los alumnos en la creación de proyectos productivos.

Es evidente entonces que los proyectos productivos y el emprendimiento tienen una relación directa, por consiguiente, es imperativo que las IEM promuevan a su vez el 
emprendimiento en las aulas, con la finalidad de asegurar que los proyectos productivos que se generen se logren promover relacionado con las comunidades del área de influencia y que no se queden solo en documentos académicos, en este orden de ideas, se debe buscar la intervención con el medio externo para reconocer los problemas reales y de este modo establecer las posibles alternativas de proyectos productivos como resultado de las dinámicas académicas.

Es asíque, partiendo de lo expuesto se busca identificar las experiencias existentes y autores representativos en torno a la formulación de proyectos productivos en las IEM, con la finalidad de generar una reflexión con el trabajo investigativo realizado con ocho Instituciones educativas del Municipio de Pitalito: Winnipeg - Sede Charguayaco; La Laguna, Domingo Savio, Chillurco, Criollo, Pachakuti, Palmarito y Villa Fátima.

\section{MARCO DE REALIDAD ACTUAL: UNA MIRADA AL CONTEXTO COLOMBIANO Y REGIONAL (PITALITO)}

De acuerdo a Hoffmann, Fuglsang y Vestergaard (2012) citado por Paños (2017), "la competencia emprendedora no se puede enseñar eficazmente utilizando métodos tradicionales." (p. 41), es por ello que el emprendimiento no puede limitarse a una cátedra orientada en las aulas de clase, en este sentido debe involucrar la práctica como parte del proceso de aprendizaje y la generación de proyectos productivos como fin primordial.

Partiendo de la necesidad de conocer el contexto relacionado con la formulación de proyectos productivos desde las IEM y la sinergia que debe existir con el emprendimiento, además de la creación de empresas como alternativa de proyecto de vida para los estudiantes que se encuentran en la educación media, se realiza una indagación sobre autores que abordaron la temática relacionada como se puede apreciar en la Tabla 1. 
Tabla. 1. Investigaciones acerca de emprendimiento, creación de empresas y proyectos desde las IEM

\begin{tabular}{|c|c|c|c|c|c|}
\hline Autor & Año & Ciudad & Contexto & $\begin{array}{l}\text { Tipo de } \\
\text { educación }\end{array}$ & Enfoque \\
\hline Paños, J. & 2017 & $\begin{array}{l}\text { Bilbao, } \\
\text { España }\end{array}$ & Urbano & Presencial & $\begin{array}{l}\text { Análisis de metodologías de la } \\
\text { educación en emprendimiento }\end{array}$ \\
\hline Blas, J. \& Rojas, V. & 2015 & $\begin{array}{l}\text { Padre Abad, } \\
\text { Peru }\end{array}$ & Rural & Virtual & $\begin{array}{l}\text { Competencias de emprendimiento } \\
\text { para educación virtual }\end{array}$ \\
\hline $\begin{array}{l}\text { Santana, L., García, L. \& } \\
\text { González, O, }\end{array}$ & 2016 & $\begin{array}{l}\text { Islas } \\
\text { Canarias, } \\
\text { España }\end{array}$ & $\begin{array}{l}\text { Rural y } \\
\text { urbano }\end{array}$ & Presencial & $\begin{array}{l}\text { Caracterización de los estudiantes } \\
\text { que están interesados en crear } \\
\text { empresa }\end{array}$ \\
\hline $\begin{array}{l}\text { Luis, M., Palmero, C. \& } \\
\text { Escolar, M. }\end{array}$ & 2015 & $\begin{array}{l}\text { Castilla } \\
\text { y león, } \\
\text { España }\end{array}$ & $\begin{array}{l}\text { Rural y } \\
\text { urbano }\end{array}$ & $\begin{array}{l}\text { Presencial y } \\
\text { virtual }\end{array}$ & $\begin{array}{l}\text { Análisis de las necesidades } \\
\text { educativas } \\
\text { en relación con el emprendimiento }\end{array}$ \\
\hline Novoa, A. & 2004 & $\begin{array}{l}\text { Bogotá, } \\
\text { Colombia }\end{array}$ & Rural & Presencial & $\begin{array}{l}\text { La educación rural en colombia y } \\
\text { la enseñanza de la ejecución del } \\
\text { PPP }\end{array}$ \\
\hline Hernandez, P. & 2006 & $\begin{array}{l}\text { México, } \\
\text { México D.F. }\end{array}$ & $\begin{array}{l}\text { Rural y } \\
\text { urbano }\end{array}$ & Presencial & $\begin{array}{l}\text { Proyectos Educativos } \\
\text { Emprendedores y la formación de } \\
\text { estudiantes emprendedores }\end{array}$ \\
\hline Cifuentes, J. \& Rico, S. & 2016 & $\begin{array}{l}\text { La Palma, } \\
\text { Colombia }\end{array}$ & Rural & Presencial & $\begin{array}{l}\text { Representaciones sociales } \\
\text { de los jóvenes rurales frente } \\
\text { a los proyectos pedagógicos } \\
\text { productivos y el emprendimiento } \\
\text { en el contexto de la nueva } \\
\text { ruralidad }\end{array}$ \\
\hline $\begin{array}{l}\text { Oliver, A., Galiana, L. \& } \\
\text { Marta, G. }\end{array}$ & 2016 & $\begin{array}{l}\text { Valencia, } \\
\text { España }\end{array}$ & Urbano & Presencial & $\begin{array}{l}\text { Diagnóstico multidimensional } \\
\text { de las actitudes emprendedoras } \\
\text { en secundaria y la relación con } \\
\text { las políticas de promoción del } \\
\text { emprendimiento }\end{array}$ \\
\hline $\begin{array}{l}\text { Rico, A. \& Santamaria, } \\
\text { M. }\end{array}$ & 2015 & $\begin{array}{l}\text { Bogotá, } \\
\text { Colombia }\end{array}$ & $\begin{array}{l}\text { Rural y } \\
\text { urbano }\end{array}$ & Presencial & $\begin{array}{l}\text { Estudio comparativo de los } \\
\text { procesos existentes en el campo } \\
\text { del emprendimiento en la } \\
\text { educación } \\
\text { media en Colombia y Ecuador. }\end{array}$ \\
\hline
\end{tabular}

En el estudio realizado por Santana, García la finalidad de obtener el perfil del estudiante \& González (2016), se buscaba adelantar el emprendedor para reflexionar sobre qué me"análisis de las características de los alumnos didas podrían fomentar el emprendimiento en adolescentes de la escuela secundaria, con las IEM" (p. 133), a través de este estudio es 
posible identificar la predisposición de los estudiantes para emprender y su formación para la actividad empresarial, además de identificar cómo intervienen las IEM en el desarrollo de las competencias y el espíritu emprendedor.

Adicionalmente, Santana, García \& González (2016) concluyen que:

"La educación repercute a largo plazo en las actitudes emprendedoras. La formación específica influye a corto plazo en las actitudes y prepara al alumnado a través de actividades prácticas, simuladas en el aula o reales, para la planificación, organización y ejecución de proyectos empresariales innovadores." (p. 135).

En este orden de ideas, los proyectos productivos son el resultado de un trabajo articulado por un grupo o individuo para satisfacer una necesidad o dar solución a una problemática, por lo tanto, la generación de estos proyectos deben partir desde la formación para el emprendimiento en los espacios académicos a través de prácticas en el aula pero enfocados a responder necesidades del entorno.

Tomando como referencia la experiencia anterior, es posible evidenciar que en las aulas de clase de las IEM de Pitalito, se busca el fomento del emprendimiento a través del fortalecimiento de las competencias y actitudes que favorecen la generación de la cultura de del emprendimiento con perspectiva de desarrollo sostenible, donde los estudiantes cuentan con espacios para el desarrollo de ideas innovadoras y el acompañamiento docente, en este orden de ideas, se puede entender que el espíritu emprendedor se logra potencializar a través de las actividades prácticas.

Por otra parte, Huber y Van Praag (2014) citado por Blas y Rojas (2015) mencionan que "es fundamental desarrollar emprendimiento en edades tempranas lo que significa fortalecer sus habilidades cognitivas y no cognitivas, esto a priori produce efectos indirectos haciendo de su vida más productivo." (p. 6), en otras palabras, en las aulas de clase se pueden propiciar espacios para que los estudiantes puedan mejorar sus habilidades para emprender, lo cual aporta al desarrollo de la autonomía, la autoestima, el sentido de pertenencia, la asociatividad, la cooperación y la solidaridad social en su proceso formativo, al igual que en el proyecto de vida.

Es por ello, que para continuar contribuyendo al desarrollo de las economías en las regiones, es necesario seguir mejorando las condiciones de los jóvenes desde las IEM en materia de emprendimiento, siendo estas las edades propicias para fortalecer el potencial investigativo e innovador de los estudiantes. Por 
tal motivo se hace necesario analizar también la implementación de alternativas metodológicas para el fomento del emprendimiento en la educación media, como lo es la metodología virtual donde Blas y Rojas (2015) proponen "el desarrollo de competencias emprendedoras a través de una prueba piloto en entornos virtuales de aprendizaje y el desarrollo de una feria de proyectos emprendedores" (p. 32), de esta manera, se propone fortalecer el emprendimiento apoyado en las Tecnologías de la Información y la Comunicación-TIC con los entornos virtuales de aprendizaje, sin excluir el acompañamiento docente como mediador del proceso de formación, además de buscar la ejecución de ideas de proyecto como resultado final.

A través del estudio realizado por Blas y Rojas (2015), "se implementó una plataforma Moodle evidenciando de forma positiva el desarrollo de la competencia emprendedora de los estudiantes; al finalizar la experiencia, los estudiantes organizados en grupos presentaron propuestas productivas con soluciones novedosas a problemas de su entorno." (p. 69), también, se puede mencionar que la virtualidad es una de las estrategias metodológicas válidas para el fomento del emprendimiento, de igual importancia que los espacios presenciales, donde también se debe garantizar que los estudiantes puedan desarrollar su espíritu emprendedor hasta el punto de materializar sus ideas en acciones, generando proyectos productivos desde el aula.

Del mismo modo se puede mencionar, lo expuesto en la investigación realizada por Paños (2017) donde concluye que "las metodologías son el vehículo para desarrollar las competencias. Independiente de la competencia que se quiere trabajar, estas deberían ser activas, centradas en el estudiante, participativas, donde se les ofrezca a los alumnos múltiples situaciones contextualizadas y variadas." (p.44), ciertamente, se demuestra que la metodología puede ser implementada de acuerdo a las condiciones y particularidades de la IEM, sin que esto cambie el fin principal de fomentar el emprendimiento y lograr la formulación de proyectos productivos.

Por su parte, Osorio y Pereira (2011) mencionan que:

"la educación tradicional en emprendimiento centra su esfuerzo en dos aspectos. Por un lado, da herramientas y habilidades a los estudiantes para identificar mercados, oportunidades y recursos; por otra, enseña la gestión de las nuevas empresas. Pero no hay una formación adecuada sobre los aspectos próximos y distantes que influyen en los pensamientos, expectativas y creencias del individuo." (p. 22). 
Es decir, que la educación emprendimiento, debe buscar la integralidad y flexibilidad del currículo, fortaleciendo el propósito formativo de los estudiantes y permitiendo que los docentes puedan evaluar de manera permanente las prácticas desarrolladas en el aula, de tal forma que sea posible el rediseño de las estrategias didácticas, para lograr que al estudiante se le pueda brindar una formación de calidad para incentivar el espíritu emprendedor. Justamente, Hernandez (2006) considera que:

"Los recursos didácticos que se emplean están seleccionados, adaptados o construidos con la finalidad de facilitar la reflexión, esclarecer la problemática a la que se enfrentan los estudiantes; o servir como guía para la construcción del sistema explicativo, del prototipo construido, o del producto elaborado." (p. 69).

Una vez entendido el fomento del emprendimiento como proceso necesario para generar proyectos productivos en los estudiantes de Educación Media y las alternativas metodológicas que en definitiva establecen mecanismos similares en el proceso de aprendizaje pero que buscan el mismo fin, se hace necesario comprender porque desde las IEM es posible generar cambios significativos en sus comunidades, teniendo en cuenta lo mencionado, en el estudio de Luis, Palmero \& Escolar (2015), plantean como objetivo:
“Determinar el impacto que la educación tiene en las tasas de emprendimiento, como uno de los factores que definen las interacciones sociales, económicas, políticas y éticas de un país, motivando cambios en los procesos de socialización e innovaciones curriculares que deben operarse en la formación de los jóvenes emprendedores en una sociedad de redes." (p. 225).

De este modo, se puede decir que el papel de las instituciones de educación media IEM es de gran relevancia para mejorar los índices en el emprendimiento en las naciones, en espera de que sea posible intervenir en las comunidades con proyectos productivos que logren dinamizar las economías de las regiones. Asimismo, el rediseño curricular y la actualización del PEI son esenciales para establecer criterios que se ajusten a la realidad.

Por consiguiente, Luis, Palmero \& Escolar (2015) concluyen que:

"Aunque exista un gran debate en torno a las interacciones entre educación y emprendimiento, que el estudio realizado permite afirmar que el currículo es el instrumento idóneo para la adquisición de competencias relacionadas con la creación de empresas, y los resultados obtenidos del grupo de discusión reclaman una revisión del currí- 
culo de la educación secundaria que debe configurarse como la institución en la que se aprende a compartir, dialogar y adquirir conocimientos técnicos, especialmente se subrayan los que guardan relación con idiomas, nuevas tecnologías, legislación y habilidades sociales de comunicación, liderazgo, adaptación al cambio, responsabilidad y trabajo en equipo." (p. 245).

En efecto, estos resultados indican lo ya mencionado por la ley 1014 de 2006 referente a la articulación del emprendimiento con el PEI y a su vez la necesidad de rediseñar el currículo con la finalidad de generar nuevas posibilidades para la formación emprendedora de los estudiantes.

Ante lo expuesto, se puede evidenciar que en las IEM de Pitalito se encuentran fomentando el emprendimiento con sus estudiantes, a través de actividades en las aulas, favoreciendo el proceso de aprendizaje para adquirir conocimientos y capacidades para planear gestionar procesos, innovar, crear, inventar y cuidar el ambiente, sobre este último, se evidencia que en la actualidad están desarrollando los Proyectos Ambientales Escolares-PRAE, que “incorporan la problemática ambiental local al quehacer de las IEM, teniendo en cuenta la dinámica natural y socio cultural del contexto con la Formación para: Ser - saber y saber para el manejo sostenible del ambiente." Ministerio de Ambiente, Vivienda y Desarrollo Territorial (2012), en este sentido, se está generando una experiencia significativa en la articulación de proyectos con el PEI en búsqueda de incorporar propuestas pedagógicas en el diseño curricular.

Sobre todo, las IEM de Pitalito deben procurar que las experiencias recogidas de los PRAE, sean tenidas en cuenta como punto de referencia para iniciar con la articulación de los PPP y de este modo comenzar a incluir las propuestas en el diseño curricular, dando respuesta a las necesidades del contexto. Sobre lo mencionado, Paños (2017) considera que “las instituciones educativas no deberían quedarse pasivas y deben adaptarse a las nuevas demandas del siglo XXI respondiendo ante los cambios." (p. 44).

Siendo consecuentes con lo afirmado por Hernández (2006), donde considera que "Las iniciativas emprendedoras se hacen desde los principios establecidos en los programas de estudios, a partir de la filosofía adoptada por el centro educativo, o partiendo de las necesidades manifiestas de la población." (p. 61), es evidente, que el trabajo que se debe desarrollar en las instituciones debe estar inmerso en los PEI como iniciativas que se generen desde las instituciones en búsqueda de la transformación para el 
desarrollo económico de la región, al igual que ajustar las prácticas pedagógicas para educar a los estudiantes de acuerdo a las nuevas competencias requeridas en el contexto global.

En cuanto a la necesidad de ir más allá de lo expuesto, se puede continuar con el análisis de las experiencias de la articulación de los PPP en la educación media, como caso típico se revisa la investigación adelantada por Cifuentes y Rico (2016), donde realizan la “Caracterización de las representaciones sociales de estudiantes de los grados décimo y once, frente a los proyectos pedagógicos productivos y el emprendimiento en el contexto de la nueva ruralidad en una IEM de La Palma Cundinamarca." (p. 92), sobre esta experiencia, se logra evidenciar que los jóvenes consideran que a través de la educación pueden aprender a desarrollar proyectos productivos y a su mejorar las prácticas desde la ruralidad, lo que conlleva a generar un proyecto de vida desde la región mejorando las condiciones técnicas y productivas.

Cabe destacar, que la investigación de Cifuentes y Rico (2016), recomienda que desde "La innovación educativa se contribuya en la cualificación y pertinencia de la práctica pedagógica en el desarrollo de los proyectos pedagógicos productivos y en la formación para el emprendimiento, desde la perspectiva de los estudiantes." (p. 101). Cabe mencionar, que la iniciativa de los PPP es una responsabilidad de las IEM, pero a su vez es importante que se articulen todos los actores para contribuir a una estructura que fortalezca la cultura del emprendimiento, además es importante considerar, los aportes de los jóvenes quienes son los llamados a definir las ideas innovadoras partiendo desde la creatividad y las necesidades que reconocen en la región.

Desde las experiencias recogidas en el desarrollo del proyecto investigativo con las IEM de Pitalito, se encuentra la necesidad de que las actividades de emprendimiento se articulen con la educación superior para adelantar la capacitación docente en el fortalecimiento de herramientas metodológicas y prácticas docentes para transformar de las iniciativas de emprendimiento en proyectos productivos; La articulación propicia la generación de rutas formativas para el fomento del emprendimiento y la formulación de estrategias para fortalecer la relación con el sector productivo.

\section{CONCLUSIONES}

Para lograr la formulación de proyectos productivos es necesario mencionar el emprendimiento como eje estratégico para fomentar el espíritu emprendedor de los estudiantes, en otras palabras, no se puede hablar de proyectos productivos si no existen lineamientos claros 
referente al desarrollo del emprendimiento desde las aulas, teniendo en cuenta que unos consecuencia del otro.

Las IEM cuentan con un potencial bastante importante para el fomento al emprendimiento, teniendo en cuenta que los estudiantes en edades tempranas tienen una visión creativa e innovadora, sin embargo es necesario continuar con la motivación desde las aulas de clase motivando a los estudiantes para que la formulación de proyectos productivos trascienda las fronteras de la institución educativa para aportar al desarrollo de la región y de igual manera sean una alternativa de proyecto de vida.

Indiscutiblemente el PEI es el documento que orienta los lineamientos para el fomento del emprendimiento, su contenido debe vincular el sentir de la comunidad académica y las necesidades de las regiones desde lo productivo, justamente las IEM de Pitalito deben realizar un proceso de resignificación del $\mathrm{PEI}$, con la finalidad de actualizar el currículo de acuerdo a las tendencias del entorno globalizado y las particularidades de la región.

Los PPP son propuestas que nacen a partir de las necesidades identificadas en la región y deben tener una sinergia con lo definido en el PEI, por esta razón, las IEM de Pitalito deben adoptar los PPP para estructurar de manera organizada los procesos de planeación, ejecución y evaluación de las prácticas proactivas que se generan en el aula.

El docente influye de manera directa en la motivación de los estudiantes, por lo tanto, el proceso de acompañamiento debe ser ameno, donde se brinden espacios de reflexión para que el estudiante fortalezca sus habilidades y competencias para la generación de ideas innovadoras, además, la cualificación docente debe ser permanente para mejorar las prácticas pedagógicas y los recursos didácticos dispuestos en las aulas.

Le MEN tiene definidas cartillas y manuales para el fomento del emprendimiento y la estructura de los PPP, en este orden de ideas, el reto de las IEM de Pitalito radica en fortalecer el diseño curricular en pro de generar experiencias productivas desde las aulas con la intencionalidad de tener mayor apropiación de la cultura del emprendimiento.

En la metodología virtual o presencial, se establecen actividades similares aunque difieren en el uso de algunos recursos pero tienen el mismo propósito formativo, es decir, que el fomento del emprendimiento se puede enseñar a partir de diferentes metodologías y no existe claridad frente a la mejor opción, sin embargo el proceso de evaluación de las prácticas 
utilizadas debe ser evaluado de forma permanente, con la finalidad de lograr que se cumpla con los propósitos formativos para dinamizar la formulación de proyectos productivos.

\section{REFERENCIAS}

Blas \& Rojas (2015). Uso de la plataforma Moodle para el desarrollo de la competencia de emprendimiento en los estudiantes del $4^{\circ}$ grado de educación secundaria de menores de una institución educativa de Ucayali, [Tesis]. Pontificia Universidad Católica del Perú, Escuela de Posgrado. Perú: San miguel. Recuperado de http://tesis.pucp.edu.pe/repositorio/handle/123456789/7044

Cifuentes, J. \& Rico, S. (2016). Proyectos pedagógicos productivos y emprendimiento en la juventud rural. Zona Próxima, (25), 87-102.

Colombia (2006). Ley 1014 de 2006 - De fomento a la cultura del emprendimiento. Bogotá D.C.: El Congreso de la República de Colombia.

Hernández, P. (2006). Proyectos Educativos Emprendedores. Ediciones Ángeles Hermanos. México: México D.F,

Luis, M., Palmero, C. \& Escolar, M. (2015). Impacto de la educación en el emprendimiento. MAKINGOF y análisis de tres grupos de discusión.
Pedagogía Social. Revista Interuniversitaria, 2015, 25, (p. 221-250)

Ministerio de Ambiente, Vivienda y Desarrollo Territorial (2012). Guia de diseño e implementación de proyectos ambientales escolares PRAE desde la cultura del agua. Bogotá D.C.: Colombia. Recuperado de http://www.minambiente.gov. co/images/GestionIntegraldelRecursoHidrico/ $\mathrm{pdf} / \mathrm{cultura-del-agua/Guia-de-diseno-e-}$ implementacion-de-PRAE-desde-la-culturadel-agua.pdf

Ministerio de Educación Nacional-MEN (2010). Proyectos Pedagógicos Productivos: Una estrategia para el aprendizaje escolar y el proyecto de vida. Parte 1. Bogota D.C. Recuperado de https://www.mineducacion.gov.co/1759/ articles-287836_archivo_pdf.pdf

Ministerio de Educación Nacional-MEN (2010). Proyectos Pedagógicos Productivos: Una estrategia para el aprendizaje escolar y el proyecto de vida. Parte 2. Bogota D.C. Recuperado de https://www.mineducacion.gov.co/1759/ articles-287836_archivo_pdf_parte2.pdf

Ministerio de Educación Nacional-MEN (2012). La cultura del emprendimiento en los establecimientos educativos: Orientaciones generales. Guia No. 39. Bogota D.C. Recuperado 
de https://www.mineducacion.gov.co/1759/ articles-287822_archivo_pdf.pdf

Novoa, A. (2004). Educación y producción en el desarrollo rural, una innovación metodología en el caso de Colombia. Ponencia para el I ForoTaller "Alternativas para la educación rural en Venezuela. Tarabana, Edo. Lara. Venezuela.

Oliver, A., Galiana, L. \& Gutiérrez, M. (2016). Diagnóstico y políticas de promoción del emprendimiento en estudiantes. Anales de Psicología, 32 (1), 183-189.

Osorio, F. \& Pereira, F. (2011). Hacia un modelo de educación para el emprendimiento: una mirada desde la teoría social cognitiva. Cuadernos De Administración, 24(43).
Paños, J. (2017). Educación emprendedora y metodologías activas para su fomento.

Revista Electrónica Interuniversitaria de Formación del Profesorado, 20 (3), 3348.

Quevedo, R. (2005). La educación y la capacitación rural en la región andina. Agroalimentaria, 11(21), 93-112.

Rico, A. \& Santamaria, M. (2018). Análisis comparativo de los procesos existentes en el campo del emprendimiento en la educación media en Colombia y Ecuador. Voces y Silencios: Revista LatinoamericanadeEducación,Vol.8,No.2,53-68 Santana, L., García, \& L. González, O. (2016). Emprendimiento y adolescencia. NEW APPROACHES IN EDUCATIONAL RESEARCH. Vol. 5. No. 2. Julio (p. 131-138). ISSN: 2254-7339 